\title{
De Calore Solis, a Treatise on Heat by Robert Grosseteste
}

\section{Amelia Carolina Sparavigna'}

\author{
${ }^{1}$ Department of Applied Science and Technology, Politecnico di Torino, Italy
}

\begin{abstract}
In this paper we are translating and discussing one of the scientific treatises written by Robert Grosseteste, the De Calore Solis, On the Heat of the Sun. In this treatise, the medieval philosopher analyses the nature of heat.
\end{abstract}

Keywords: History of Science, Medieval Science

\section{Introduction}

Robert Grosseteste (c. 1175 - 1253), philosopher and bishop of Lincoln from $1235 \mathrm{AD}$ until his death, wrote several scientific papers [1]; among them, quite remarkable are those on optics and sound [2-4]. In [5], we have recently proposed a translation and discussion of his treatise entitled De Impressionibus Elementorum, written shortly after 1220; there, Grosseteste is talking about some phenomena involving the four classical elements (air, water, fire and earth), in the framework of an Aristotelian physics of the atmosphere. This treatise on elements shows the role of heat in phase transitions and in atmospheric phenomena. Here we will translate and discuss another Grosseteste's treatise, that entitled De Calore Solis, on the Heat of Sun.

The study of heat and related phenomena has a long history. The first to have proposed a theory on heat was Heraclitus, the Greek philosopher who lived around the 6th century BCE in Ephesus. He is wellknown for his proverbial "Panta rhei", all things are flowing. According to Heraclitus, the four classical elements of nature are subjected to transformations, which are the replacement of one element by another: "The death of fire is the birth of air, and the death of air is the birth of water." [6] He saw the fire as the central element, controlling and modifying the others elements. The universe was in a continuous state of flowing as a result of transformations caused by the fire.

The ancient Greek philosophy imagined the universe created by four elements then. Quite probably, Plato was the first to use the term "element", "stoicheion", in reference to air, fire, earth and water. The Greek word "stoicheion" is meaning "smallest division" or "syllable", the smallest unit used to create all the words; therefore, by analogy, all the things are composed of elements such as the words of syllables. Moreover, according to Aristotle, the elements have some features: he tells that Air is wet and hot, Fire is hot and dry, Earth is dry and cold, and the Water is cold and wet [5]. And, because the above-mentioned four elements are corruptible, Aristotle added the incorruptible Aether as the fifth element, or essence, the quintessence, and since we do not see any changes in the heavenly regions, the stars and skies above the Moon must be made of it.

The Hellenistic period, a period between the death of Alexander the Great in $323 \mathrm{BC}$ and the emergence of ancient Rome, saw the flourishing of science. In the Egypt of this Hellenistic tradition, under the Roman Empire of the first Century CE, Hero of Alexandria invented a steam-powered device [7]. However, it was only in the late 18th century that heat engines, having the steam as working fluid, were developed and used on a large scale. The Hellenistic cultural and scientific tradition continued in the Arabic world. In the Western Europe, after the fall of its part of the Roman Empire, it started a decline of the knowledge of Greek, and so the Christian Western Europe was cut off from an important source of ancient learning.

Although some scholars maintained some spirit of scientific inquiry, the Europe saw a certain stagnation. However, during the High Middle Ages, a period starting from the 11th century, the West Europe had begun to reorganize its scientific thought. This was stimulated by the fact that some of the ancient works of the Greek philosophers re-entered the West through translations from Arabic to Latin [8]. Their commentaries of the ancient philosophers had a great influence on medieval scholars, who mixed them with the Christian theology. Eventually, Aristotle was claimed as the greatest thinker of the ancient world, and the Aristotelian physics became the physics mostly accepted by the European Churches. 
Based on Aristotelian physics, things are moving according to their essential nature, that is, according to the elements in them. The motions in the sublunary world, that is, on Earth, were based on the tendencies of elements. Stones contained the element earth, and earthy objects tended to move naturally in a straight line toward the center of the earth, which is the center of the universe, unless otherwise prevented from doing so by some violent action. Fire has the tendency to move upwards. Celestial objects, which are made of the fifth essence, are moving in circles, because circular motion is a never ending motion and therefore perfect.

For what concerns heat in this Aristotelian framework, it was in the 11th century that al-Biruni (Abū Rayhān Bīrūnī), proposed movement and friction as causes of heat, which in turn produces the element of fire $[9,10]$. For this reason, the apparent lack of movement of the Polar Regions causes them to be cold. The earth and the water form one globe, surrounded by the atmosphere. But, the atmosphere is in contact with the sphere of the Moon, and becomes heated as a consequence of the friction between the spheres. In this manner, the fire is produced, surrounding the air. Also Avicenna says that heat is generated from motion in external things [9], whereas is generated inside in the living beings.

In the 13th century, the Islamic philosopher Abd Allah Baydawi discussed the heat in the same manner. Hot and cold are among the most obvious and plain sense perceptions. Heat has the specific ability to separate different elements and to join elements that are similar, for instance, when boiling water to vapor. The heat is then producing flux and circulation, and melting, according to the coarseness of the thing (if we read the Grosseteste's treatise on the Elements, we find there a reference to the coarseness too, [5]). Baydawi considered a natural and an artificial heat, and that the artificial heat may occur through motion-change, the proof of this being through experiment [11].

In reading the treatise on the heat of the Sun, we will find that Grosseteste is invoking the motion as a source of heat too. Here in the following the translation of his treatise from the Latin text [12].

\section{On the Heat of the Sun}

Since our main aim here is the discussion of the heat of the Sun, let us first of all inquire, in general, what is the principle of generation of heat. That is, how many and what are the principles of heat generation. Since they are three, that is, hot bodies, motions and concentrations of rays, it is better to know that in these three cases there is a heat of a single nature involved (univocal heat [13]). This univocal heat produces a univocal effect. When, therefore, there is a univocal undergoing effect, we shall find a univocal cause: in fact, every univocal effect has a univocal cause.

It is clear that the heat in all these things is univocal: because heat generated in any of these manners has the same power, makes the same accomplishment. Let us stress that this is not stated in an equivocal sense, open to more than one interpretation, but in a univocal sense. Therefore, let us search this univocal cause! In all of them, the immediate cause of heat is disgregation. Hence then, a hot thing generates heat, doing it by the disgregation of its matter. In what way, however, this disgregation is occurring in movement and in condensation of rays, it is difficult to see.

Now, the local motion, from which heat is generated, is divided into natural motion and violent motion. The natural motion is divided into straight motion and circular motion. First, we speak about the violent motion, that is, about a heavy body violently moved. It is referred that a heavy body is violently moved in three ways: up, down, or down, however not directly to the center (of the Earth). In all these cases, in a violent motion, it appears that a disgregation exists as a result of the motion. Indeed, in a violent motion there is a two-fold power, that is, natural and violent, which moves every part of the mobile in different directions. Then, from this tendency to different directions follows disgregation. And thus, as a result of the violent motion, it is necessary that what is moving is disgregated according to its parts, and thus we have heat. And because in the first of the violent motions (upwards), there is the greatest opposition in the inclinations of the two motive powers, because they tend to move in opposite directions at all, we have as a consequence the largest disgregation and heat; in the second and in the third motion, however, the generation of heat is moderate. And this is very clear from the use of reason and experiment.

The same thing is clear in the natural motion. For, heat is generated in the motion during which an object is naturally moving downwards. There is a twofold power, and accordingly a twofold force, namely natural and violent, that actually moves every part of the object. The fact that there is a natural force is evident. But we can prove that there is a violent force too: any object that is heavy and moving downwards, but not towards the center of the Earth, is moved violently. All the parts of the body do not move downwards directly towards the center of the Earth. Therefore, all the parts of the body move violently.

I prove the minor: the parts of a heavy body always keep the same distance (from each other) in general. Since, then, when they are moved downwards as a whole, they are moved on parallel lines, that is, on 
lines that are equidistant. Moreover, the equidistant lines, extended to infinity, from one part or the other, will never meet. Therefore the parts of the body that moves naturally downwards, move on lines which never meet, therefore, they do not move towards the center of the Earth, because if they moved directly to the center, they would move on lines running together, converging towards the same center. Thus, it is obvious the following principle, that, accordingly, on each part of the body moving naturally downwards, there is a two-fold force (a natural force and a violent force), tilting to different directions. However, the opposition between these two forces is small compared with the great opposition of stresses among the parts of a body in violent motion. And therefore, among all the motions which are generating heat, in the natural motion we have that the minimum amount of heat is generated. From these arguments, it is clear that there is only one cause generating heat from the natural straight motion and from the violent motion and heat from a hot body.

In the third (generation of heat) too, it is clear this similarly: that, indeed, from a collection of rays some heat is generated by the univocal cause of heat. This is clear according to "Of the Mirrors", where it is told that some tinder is ignited by a concave mirror placed at Sun: and this happens because of a disgregation. In fact, a ray in a dense transparent medium is more incorporated than in a subtler medium; we are not telling of a complete incorporation as it happens in heat, but a slight incorporation of it. However, due to this incorporation, the ray is dragging some air along; that is, when the rays are collected in the same point, at this very point, where each ray is coming along its straight pattern, we have a large dispersion of air in different directions; and so it will be disgregation and, as a consequence, heat. Then, it is clear that in these three kinds of hot there is a univocal cause.

Let us consider now the heat generated by the Sun. If, then, the Sun is generating heat, it will be as a hot body generates heat, or as motion generates heat, or as a concentration of rays generates heat. That the Sun does not generate heat as heat is generated by a hot body is clear from the following: it was proved in VII book of Physics by Aristotle, that it is necessary that the agent which is producing an alteration and the subject which is to be altered need to be in immediate contact. Therefore, if there were a medium between the first, which is producing an alteration, and the last, which is altered, it would be necessary that this medium were previously altered by the heat of the hot Sun, before altering the last subject, otherwise the abovementioned "alterans" and "alteratum" would not be in immediate contact.
Therefore, being between the Sun and air several media, and, nearest the Sun, which is altering by means of its heat, there is the quintessence or a portion of quintessence, it is necessary that this fifth element be previously altered by the heat of the hot Sun, before the air being altered. But this is impossible, because if it were alterable, it would be corruptible. Therefore the first manner is impossible, that is, it is impossible the Sun heats in the manner that a hot body generates heat.

Somebody could tell by chance that the heat in the Sun is virtually hot as the pepper is. But there is not agreement. Because the hot of the pepper is virtual not actual, it does not move, it cannot be moved, it cannot alter or being altered. And the same it would be for the Sun. But this is impossible: therefore the former is false.

That the heat is not generated from the motion of the Sun is clear too. In the same manner, the motion does not generate heat, unless in each part of the moving body there are some different tendencies to move the parts in different directions. But in everything that is moved circularly and not violently, any chosen part of it has the same inclination of the whole and there are not differences: each part has the inclination to move on a circular motion. Therefore from the circular motion it is impossible to generate heat. But, perhaps, you would say that the cause of the heat is not inside of what is moved in a circular trajectory, but it is from the outside, just as it is in the lower world from the resistance of the medium. This, however, has a twofold falsehood: one is because in the lower bodies, the resistance of the medium is not the source of the heat produced during motion [14]. Because, if it were so, it would be that, because the medium can offer equally resistance to that which is moved naturally, and to that which is forced to move, heat would be equally generated in the violent motion and in the natural motion. But this is false, as it is clear by experience: and therefore the former is false too. The other reason why it is false is in the fact that the Sun and the other stars, when moving, do not have resistance, because they are not moving by their own movements, but, since they are fixed in their own spheres, they are moving through the motion of their spheres like a ship in a river, which is moved by the motion of the river, as the Philosopher proves in his book about heavens and world.

The only possibility which remains is that the Sun generates heat through concentration of rays. This is clear: the rays of the Sun are, to some extent, incorporated in the transparent volume of the air, which is naturally dense; but the rays of the Sun falling on the surface of earth, which can be plane or concave or convex, are reflected at equal angles, as it is shown by the last of the principles in the book "On 
the Mirrors". Then, if the rays fall perpendicularly, they are reflected perpendicularly; and for this reason the ray are falling and reflected along the same path, in the completely opposite direction, and there is the largest disgregation; this happens at latitudes of the equator (Grosseteste calls it the equinoctial circle [15]), when the Sun is passing through the Zenith of these regions, and in the places declining from the equator, towards south or north, which have a latitude less that the latitude of the tropic of Cancer or less than that of the tropic of Capricorn, in the other hemisphere.

And then it is necessary, in those places, that the rays of the Sun twice a year fall perpendicularly upon them. In a place, however, the latitude of which is equal to the latitude of the tropic of Cancer or of Capricorn, it is necessary, that the Sun reaches the Zenith once a year and once a year it sends its rays perpendicularly to these places, and then, when this happens, in those places we have the largest disgregation and the highest temperatures. This is a violent disgregation, which is usually made out from a collection of rays refracted through a spherical body, or reflected from a concave mirror, but in these cases, the rays are not deviated in the completely opposite direction.

But in the climatic zones, where the latitude from the equator is greater than the tropic of Cancer, in northern regions then, because the Sun does not reach the Zenith, the rays fall at angles smaller than the right angle and are reflected according to them, therefore not reflected in the totally opposite direction. And the more distant the place is from the equator, the more obtuse the angles at which the rays fall and are reflected and the less the disgregation and heat generated. This is also shown by the experience.

If, however, it is asked, why heat is not generated in the fifth essence from the rays of the Sun, it is possible to reply in two manners: first, because they do not intersect themselves after reflection; moreover, even if they intersect after reflection in the totally opposite direction, they do not generate heat or warmth. For, since this transparent medium does not possess a dense nature, the solar rays are not in any way incorporated in it, and then they cannot disgregate in any way the parts of matter. And so, also in the highest layer of air, where the air is rarefied, the heat is minimally generated, as shown by observations. There is plenty of snow on the top of mountains, where the solar rays are brighter than in the valleys, and there is the reflection of rays as in the valleys too; however, because of a lower density of air there, the atmosphere has a smaller density and therefore a little incorporation of light with air, and a small disgregation of parts of air when rays are collected. Here, (at sea level), we have a larger incorporation of rays and as a consequence a greater disgregation and heat. The end.

\section{Discussion}

Other published translations of De Calore Solis are at $[14,16,17]$.

In the first part of this treatise, Grosseteste is talking of the phenomenon of disgregation, "disgregatio" in the Latin text, as the source of heat. The Latin "disgregatio" is the dispersal of an object in several parts and then a process of separation. In the translation, I used "disgregation" because it was used by Clausius too, instead of the term "scattering" used in [14]. Between 1850 and 1865, the German physicist Rudolf Clausius, had the necessity to find some terms useful for the new science of thermodynamics. He used the "caloric", introduced in 1780, by Antoine Lavoisier, for the substance of heat. In 1862, Clausius linked the integral of $\mathrm{dQ} / \mathrm{T}$, the ratio of heat exchange and temperature, to something he called "disgregation" of the body, having relation to the arrangement of molecules of the working body. This happened after reading the Carnot's 1824 paper "On the Motive Power of Fire" that characterized the transformations of working substances in an engine cycle. In $1865, \mathrm{dS}=\mathrm{dQ} / \mathrm{T}$ was defined as the transformational content of the working body and then it became the entropy, so to have similarity to the word energy. The term "entropy" is coming from the German "entropie", from Greek "entropia", a turning toward, from "en", in, and "trope", a turning [18].

Going back to his treatise, let us stress that Grosseteste is proposing that the heat we observe in several phenomena is the same physical quantity. Heat from hot bodies, motions and rays of light is produced according to the same principle of disgregation. In the case of the heat produced by the rays of light, it is due to the light incorporated in a medium. In [19], A.C. Crombie explains the Grosseteste's theory in the following way: Grosseteste "concluded that all hot bodies generated heat by the scattering of their matter and that the sun generated heat on the earth in direct proportion to the amount of matter incorporated from the transparent medium (air) into its rays". In this framework, Grosseteste ends the treatise with a discussion on the climatic regions of the earth, according to the inclination of the rays of Sun. Geography and astronomical science are the subject of another treatise, the De Sfera [20], written according to the Scholastic astronomy.

The incorporation of light in matter is used by Grosseteste also in discussing the colours [19,21]. In fact, to the philosopher, colours appear when the light is mixed with a transparent medium, according to the purity of it and the brightness and intensity of rays. 
When causing the heat, the rays of light are incorporated by air. This conclusion was probably argued by Grosseteste after observing the scattering of light by the particles of dust and the consequent visibility of their motion.

\section{References}

[1] N. Lewis, Robert Grosseteste, The Stanford Encyclopedia of Philosophy, 2010.

[2] A.C. Sparavigna, On the Rainbow, a Robert Grosseteste's Treatise on Optics, The International Journal of Sciences, 2013, Volume 2, Issue 9, Pages 108-113.

[3] A.C. Sparavigna, Robert Grosseteste and his Treatise on Lines, Angles and Figures of the Propagation of Light, The International Journal of Sciences, 2013, Volume 2, Issue 9, Pages 101-107.

[4] A.C. Sparavigna, The Generation of Sounds According to Robert Grosseteste, The International Journal of Sciences, 2013, Volume 2, Issue 10, Pages 1-5.

[5] A.C. Sparavigna, Robert Grosseteste and the Four Elements, The International Journal of Sciences, 2013, Volume 2, Issue 12, Pages 42-45.

[6] The Art and Thought of Heraclitus: A New Arrangement and Translation of the Fragments with Literary and Philosophical Commentary Cambridge University Press, Sept. 3, 1981.

[7] A.C. Sparavigna, Water, Air and Fire at Work in Hero's Machines, Archaeogate, 2011, January 24.

[8] A.C. Sparavigna, From Rome to the Antipodes: The Medieval Form of the World, International Journal of Literature and Arts, 2013, Volume 1, Issue 2, Pages 16-25.

[9] Vv.Aa., Wikipedia, wikipedia.org/wiki/History_of_heat [10] A.C. Sparavigna, The Science of al-Biruni, The International Journal of Sciences, 2013, Volume 2, Issue 12, Pages 52-60.

[11] Vv.Aa., Nature, Man and God in Medieval Islam, Islamic Philosophy Theology and Science, Texts and Studies, Edited by H. Daiber and D. Pingree, Volume XLV, Brill, 2002.
[12] The Latin text is that given at "The Electronic Grosseteste", www.grosseteste.com

[13] In the Latin text, Grosseteste defines the heat as "univocus", and therefore I translated it as "univocal", in the sense of "being of a single kind".

[14] In the Latin text, there is the term "ageneratix", which seems quite odd. I translated it as "source". See also, A.C. Crombie, Science, Optics and Music in Medieval and Early Modern Thought, Continuum International Publishing Group, 1990.

[15] The "equinoctial circle" is the great circle on the celestial sphere midway between the celestial poles. Then we have the two tropical circles. One of them is called the Tropic of Cancer. When this circle was named, the Sun passed through the Zenith at its latitude, when was also in the direction of the constellation Cancer (Latin for crab). However, this is no longer true due to the precession of the equinoxes. The word "tropic" comes from the Greek "tropei", meaning "turn", referring to the fact that the Sun appears to "turn back" on the solstices.

[16] G. Kish, A Source Book in Geography, Harvard University Press, 1978.

[17] R.C. Dales, The Scientific Achievement of the Middle Ages, University of Pennsylvania Press, 1973.

[18] P.M. Harman, Energy, Force and Matter: The Conceptual Development of Nineteenth-Century Physics, Cambridge University Press, Apr 30, 1982.

[19] A.C. Crombie, Grosseteste, in the Dictionary of Scientific Biography, Scribner, 1981, Pages 548-554.

[20] M.F. Dowd, Astronomy and Compotus at Oxford University in the Early Thirteenth Century: the Works of Robert Grosseteste. Dissertation, Notre Dame, Indiana, June 2003, available at www3.nd.edu.

[21] A.C. Sparavigna, Grosseteste's Colours, arXiv:1212.6336, arXiv, 